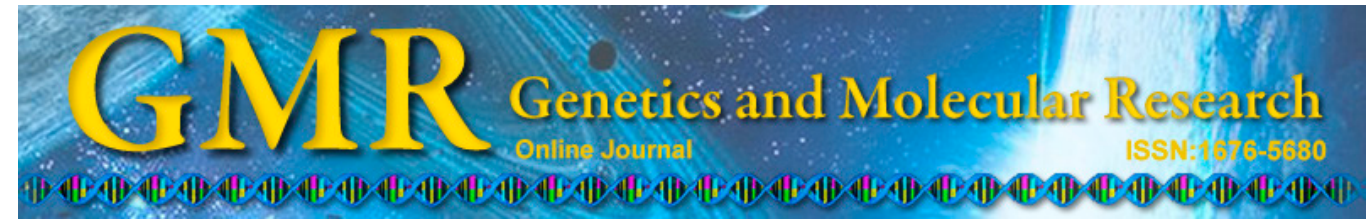

\title{
Molecular characterization of thioredoxin-1 and thioredoxin reductase activity in mud crab Scylla paramamosain
}

J.H. Hu ${ }^{1,2 *}$, F.Y. Zhang ${ }^{1 *}$, K.J. Jiang ${ }^{1}$, Y.B. Fang ${ }^{1,2}$, J. Wang ${ }^{1,2}$, M. Zhao ${ }^{1,2}$, Z.G. Qiao ${ }^{1}$ and L.B. Ma ${ }^{1}$

${ }^{1}$ Key Laboratory of East China Sea \& Oceanic Fishery Resources Exploition and Utilization, Ministry of Agriculture,

East China Sea Fisheries Research Institute,

Chinese Academy of Fishery Sciences, Shanghai, China

${ }^{2}$ College of Fisheries and Life Science, Shanghai Ocean University,

Shanghai, China

*These authors contributed equally to this study.

Corresponding author: L.B. Ma

E-mail: malingbo@vip.sina.com

Genet. Mol. Res. 13 (4): 10241-10255 (2014)

Received January 10, 2014

Accepted June 4, 2014

Published December 4, 2014

DOI http://dx.doi.org/10.4238/2014.December.4.19

ABSTRACT. The thioredoxin (Trx) system consists of thioredoxin reductase (TrxR), Trx, and nicotinamide adenine dinucleotide phosphate (NADPH). TrxR is an NADPH-dependent oxidoreductase. Trx is a ubiquitous small protein with a redox-active disulfide bridge that plays important regulatory roles in some vital metabolic reactions. In this study, a cDNA sequence $(\operatorname{Sp} \operatorname{Tr} x \mathrm{l})$ showing high identity to the first $\operatorname{Tr} x$ gene was isolated from a hepatopancreas cDNA library of the mud crab Scylla paramamosain. The full-length cDNA of SpTrx 1 consisted of $672 \mathrm{bp}$ and contained a complete open reading frame of 318 bp encoding a polypeptide of 105 amino acids. Quantitative real-time polymerase chain reaction analysis revealed that $S p \operatorname{Tr} x 1$ expression was ubiquitous in various organs of $S$. paramamosain, including the 
gill, muscle, heart, hemolymph, testis, and hepatopancreas. SpTrxI expression was upregulated significantly after Vibrio parahaemolyticus challenge: it obviously rose at $48 \mathrm{~h}$ and reached the highest level at 72 h. Furthermore, TrxR activity was detected in the gill, heart, muscle, hemolymph, and hepatopancreas. The relative TrxR activity in different tissues after $V$. parahaemolyticus injection had the same tendency in each tissue $(\mathrm{P}<0.01)$ as $\operatorname{SpTr} x 1$ expression. The TrxR activity increased $2 \mathrm{~h}$ after injection, peaked at $8 \mathrm{~h}$, slowly decreased from 12 to $24 \mathrm{~h}$, and returned to normal levels at $48 \mathrm{~h}$. The consistency of the expression between the Trx transcript and TrxR activity demonstrated that Trx was closely related to TrxR in the Trx system in S. paramamosain, suggesting that it may participate in the immune system of mud crabs.

Key words: Scylla paramamosain; Thioredoxin reductase activity; Thioredoxin reductase; Thioredoxin-1 gene; Tissue expression; Quantitative real-time polymerase chain reaction

\section{INTRODUCTION}

The thioredoxin (Trx) system is a highly conserved, ubiquitous system that plays a crucial role in redox regulation of numerous cellular signaling pathways (Müller et al., 2001; Stoyanovsky et al., 2005). It consists of Trx reductase (TrxR), Trx, and nicotinamide adenine dinucleotide phosphate (NADPH).

Trx was first described in Escherichia coli as a hydrogen donor for ribonucleotide reductase that was required for DNA synthesis (Laurent et al., 1964). Trx is a redox-related protein that exists widely in prokaryotic and eukaryotic species (Holmgren, 1985) and is characterized by the highly conserved consensus sequence-Cys-Gly-Pro-Cys- (CGPC) (Matthews et al., 1992). Trx has 2 major isoforms, Trx 1 and Trx2, in different intracellular organelles. Trx 1 is important in the cytoplasm and nucleus; Trx2 exists in mitochondria (Matsui et al., 1996; Collet et al., 2003; Song et al., 2006) as a redox regulatory protein that is ubiquitously expressed in a variety of organisms. Trx 1 has been reported to play essential roles in various signaling events (Song and Roe, 2008) and to participate in numerous physiological processes, such as cell growth and apoptosis, differentiation, and proliferation, in the course of redox regulation (Powis and Montfort, 2001). Recent evidence demonstrated that Trx1 functions as a critical myocardial protective factor directly via antioxidant effects and indirectly by protein-protein interactions with key signaling molecules (Yamawaki et al., 2003). Its activity is susceptible to the posttranslational modification processes of oxidation and nitrosylation (Kondo et al., 2006). In addition, $\operatorname{Tr} x$ genes have been reported from various species and cell types, such as channel catfish (Ictalurus punctatus) (Khayat et al., 2001), human (Jin et al., 2002), and rock bream (Oplegnathus fasciatus) (Kim et al., 2011). In crustaceans, Trxl has been reported in the shrimp Litopenaeus vannamei (Aispuro-Hernandez et al., 2008) and the crabs Eriocheir sinensis (Mu et al., 2009) and Portunus trituberculatus (Song et al., 2012).

TrxR is a ubiquitous enzyme that is involved in many cellular processes such as cell growth and protection against oxidation stress. In mammals, extracellular forms of Trx also have cytokine-like effects. Mammalian TrxR has a highly reactive active site selenocysteine residue that results in a profound reductive capacity, reducing several substrates in addition to 
Trx (Nordberg and Arnér, 2001). TrxR, in conjunction with Trx, is a ubiquitous oxidoreductase system with antioxidant and redox regulatory roles.

Mud crabs (Scylla spp) are one of the most valuable marine crabs in aquaculture throughout the Indo-Pacific (Imjongjirak et al., 2007) and Indian Ocean regions (Keenan et al., 1998; Imai and Takeda, 2005); they are regarded as one of the most commercially precious species in these areas. However, many mud crabs have died from diseases associated with Vibrio parahaemolyticus, which is the most important infectious pathogen, causing vast economic losses recently. To boost the immunity of mud crabs, researchers have made great efforts to clarify the mechanisms of immunogenic proteins. This study describes the molecular cloning and characterization of a Trx superfamily member from $S$. paramamosain (designated as $\operatorname{SpTrx1)}$ and TrxR activity, and it investigates the relationship between the Trx transcript and TrxR activity.

\section{MATERIAL AND METHODS}

\section{Construction of the cDNA library and cDNA cloning of SpTrx1}

Healthy crabs weighing $200 \pm 20$ g were captured from an aquaculture farm near Shanghai, China, and sustained in a laboratory aquarium. Total RNA was isolated from the hepatopancreas of $S$. paramamosain using TRIzol Reagent (TaKaRa, Japan) following the manufacturer protocol. The quality and concentration were checked by agarose gel electrophoresis (Universal Hood II, Bio-Rad Laboratories Inc., Hercules, CA, USA) and spectrophotometry (DU800 Nucleic Acid/Protein Analyzer, Beckman Coulter). The RNA was stored at $-80^{\circ} \mathrm{C}$ until use.

A cDNA library was constructed successfully using the SMART ${ }^{\mathrm{TM}}$ cDNA Library Construction Kit (TaKaRa), which was followed by transforming, screening, and sequencing (Zhang et al., 2011).

Random sequencing was performed after cDNA library construction. All the expressed sequence tags were subjected to Basic Local Alignment Search Tool analysis. A cDNA sequence comprising the SpTrxl domain was obtained and subjected to further investigation. Rapid amplification of 5'- and 3'-cDNA ends (5'- and 3'-RACE) was performed following the manufacturer protocol using the SMART ${ }^{\mathrm{TM}}$ RACE cDNA Amplification Kit (Clontech) to obtain the full-length cDNA sequence. A gene specific-primer, SpTrx1-5GSP (5'-GCTTGTAGTGGTGGACTTCTATGCC-3', as a 5'-RACE amplification primer) was designed according to the known SpTrxl sequence using Primer Premier 5.0. Another genespecific primer, SpTrx 1-3GSP (5'-TTCACTTGCTCCTGAGAAACTGTCC-3', as a 3'-RACE amplification primer) was designed according the obtained sequence and used for 3'-RACE. Polymerase chain reaction (PCR) was performed following manufacturer instructions.

The products were checked by agarose gel, purified by the Agarose Gel DNA Purification kit Ver. 2.0. (TaKaRa), cloned into the pMD19-T vector (TaKaRa), and sequenced.

\section{Bioinformatic analysis}

General features of the SpTrx1 full-length cDNA were detected by Vector NTI Advance 11.5, and the peptide sequence was deduced according to other Trx 1 peptide sequences from the National Center for Biotechnology Information (NCBI) database using the open 
reading frame finder (available online at http://www.ncbi.nlm.nih.gov/gorf/gorf.html). The biochemical features of the deduced protein were calculated with the Expert Protein Analysis System (http://web.expasy.org/). Amino acid sequences from various species were attained from the NCBI GenBank and analyzed using the Vector NTI Suite Advance 11.5. Phylogenetic analysis was performed by the neighbor-joining method using the MEGA software version 5.0 (Saitou and Nei, 1987). The confidence probability (multiplied by 100) that the interior branch length is $>0$, as estimated using the bootstrap test (500 replicates), is shown below the branches (Rzhetsky and Nei, 1992; Dopazo, 1994). The evolutionary distances were computed using the Poisson correction method and were in the units of the number of amino acid substitutions per site. The secondary structure of the SpTrx1 protein was predicted by the PredictProtein package (available online at http://www.predictprotein.org/). The 3-dimensional structure of the SpTrx1 protein was simulated using the SWISS-MODEL server (available online at http://swissmodel.expasy.org/) (Schwede et al., 2003; Arnold et al., 2006).

\section{Tissue distribution analysis}

Healthy crabs weighing $200 \pm 20 \mathrm{~g}$ were collected from an aquaculture farm near Shanghai and sustained in a laboratory aquarium. During the experiment, 36 crabs were fed clam meat once daily, kept in the filtered aerated seawater, and divided into 3 groups. Seven days later, 3 crabs were randomly selected from each group. We collected different tissues, including hemolymph, muscle, heart, gill, testis, and hepatopancreas, from the crabs and preserved them in liquid nitrogen for RNA isolation. Among them, hemolymph samples were withdrawn by a syringe needle from the unsclerotized membrane of the crab into a syringe containing pre-cooled $\left(4^{\circ} \mathrm{C}\right)$ anticoagulant $(0.45 \mathrm{M} \mathrm{NaCl}, 0.1 \mathrm{M}$ glucose, $30 \mathrm{mM}$ trisodium citrate, $26 \mathrm{mM}$ citric acid, and $10 \mathrm{mM}$ ethylenediaminetetraacetic acid, $\mathrm{pH}$ 4.6) to reach a volume ratio of $1: 1$ and were pelleted by centrifugation for $10 \mathrm{~min}$ at $8000 \mathrm{rpm}$ and $4{ }^{\circ} \mathrm{C}(\mathrm{Al}-$ legra $^{\mathrm{TM}}$ 64R Centrifuge, BECKMAN COULTER) (Rodriguez et al., 1995; Jiang et al., 2011; Zhang et al., 2011). Total RNA was isolated from different tissues of S. paramamosain using TRIzol Reagent (TaKaRa) following the manufacturer protocol. The quality and concentration were checked as described above.

Total RNA $(1 \mu \mathrm{g})$ was reverse transcribed with the quantitative real-time PCR (qRT-PCR) Kit for the first-strand cDNA. The products were diluted 10 times and stored at $-20^{\circ} \mathrm{C}$ until qRT-PCR analysis.

The qRT-PCR assay was performed in a detection system (StepOnePlus, Applied Biosystems). The transcript expression profiles of SpTrx 1 in 6 tissues of S. paramamosain (hepatopancreas, muscle, gills, testis, heart, and hemolymph) were detected by qRT-PCR using a pair of $\operatorname{SpTr} x$ 1-specific primers (SpTrx1-RT-F: 5'-CTTTGACAAGCAGCTGAAGGAAGCT-3' and SpTrx 1-RT-R: 5'-CCTGAATCTTGGGGGAAATCATCTT-3'). A pair of $18 S$ rRNA primers (18S-RT-F: 5'-GGGGTTTGCAATTGTCTCCC-3' and 18S-RT-R: 5'-GGTGTGTACAA AGGGCAGGG-3') were designed from the $18 S$ rRNA sequence from the NCBI GenBank (GenBank accession No. FJ646616.1) and used as the internal control. SpTrxl expression levels were calculated by the standard curve method. Amplifications were performed on a 96-well plate with a $20-\mu \mathrm{L}$ reaction volume containing $10 \mu \mathrm{L} 2 \mathrm{X}$ Power SYBR Green PCR Master Mix (TaKaRa), $1.0 \mu \mathrm{L} 10 \mu \mathrm{M}$ PCR forward primer, $1.0 \mu \mathrm{L} 10 \mu \mathrm{M}$ PCR reverse primer, $2.0 \mu \mathrm{L}$ cDNA template, and $6 \mu \mathrm{L}$ diethylpyrocarbonate-treated water. The reaction process for qRT-PCR was $10 \mathrm{~min}$ at $95^{\circ} \mathrm{C}$, followed by 40 cycles of $95^{\circ} \mathrm{C}$ for $15 \mathrm{~s}$ and $60^{\circ} \mathrm{C}$ for $1 \mathrm{~min}$. 


\section{mRNA expression profile of SpTrx1 in hemolymph with Vibrio challenge}

During the experiment, 66 healthy crabs weighing $200 \pm 20 \mathrm{~g}$ were fed clam meat once daily and kept in the filtered aerated seawater and divided into 3 experimental groups and 1 control group. Live V. parahaemolyticus $\left(4.00 \times 10^{6} \mathrm{CFU} / \mathrm{mL}\right)$ was used as the infection material against the crabs. Six crabs were untreated before sampling ( $0 \mathrm{~h}$ treatment); $60 \mathrm{crabs}$ were divided into 3 experimental groups, and each group was injected with $10 \mu \mathrm{L} \mathrm{V}$. parahaemolyticus solution through the unsclerotized membranes of the right fourth appendage with a needle. Crabs in the control group were injected with $10 \mu \mathrm{L} 0.9 \% \mathrm{NaCl}$. The hemolymph of each crab was randomly sampled at $0,2,4,6,8,12,18,24,48,72$, and $96 \mathrm{~h}$ post-injection from the challenge and control groups. All collected hemolymph samples were homogenized in TRIzol reagent. The total RNA was extracted and stored at $-80^{\circ} \mathrm{C}$, and the $\operatorname{SpTrx} 1$ expression levels in the hemolymph were measured by qRT-PCR with the same primers and methods as described above.

\section{TrxR activity}

Healthy crabs weighing $200 \pm 20 \mathrm{~g}$ were obtained as above. Fifteen crabs were used to determine the TrxR activity in different tissues (gill, heart, muscle, hemolymph, and hepatopancreas); each tissue was tested 3 times. Thirty crabs were used to determine TrxR activity in different tissues (gill, heart, hemolymph, and hepatopancreas) $0,2,4,6,8,12,18,24$, and $48 \mathrm{~h}$ post-injection with $10 \mu \mathrm{L}$ V. parahaemolyticus $\left(4.00 \times 10^{6} \mathrm{CFU} / \mathrm{mL}\right)$ solution through the unsclerotized membranes of the right fourth appendage with a needle. At the same time, each tissue was also tested 3 times. TrxR activity $(\lambda \max =412 \mathrm{~nm})$ was detected by a TrxR activity colorimetric assay kit (BioVision). One unit TrxR is the amount of enzyme that generates 1.0 $\mu$ mol 5-thio-2-nitrobenzoic acid $\left(\mathrm{TNB}^{2-}\right)$ per minute at $25^{\circ} \mathrm{C}$. The oxidation of $1 \mathrm{~mol} \mathrm{NADPH}$ to NADP will generate $2 \mathrm{~mol} \mathrm{TNB}^{2-;}$; therefore, $1 \mathrm{TNB}^{2-}$ unit equals $0.5 \mathrm{NADP}$ unit in this assay kit. TrxR activity levels were calculated by the standard curve method. The standard curve reaction volume contained $0,2,4,6,8$, and $10 \mu \mathrm{L} \mathrm{TNB}^{2-}$ standard and assay buffer was added to $100 \mu \mathrm{L}$. The experimental group was divided into 2 assays: the first measurement is the total 5,5'-dithiobis-(2-nitrobenzoic) acid (DTNB) reduction, and the second one is the DTNB reduction in the presence of the TrxR-specific inhibitor. The difference between the 2 results is the DTNB reduction by TrxR. The reaction volume contained $25 \mu \mathrm{L}$ tissue homogenate, 55 $\mu \mathrm{L}$ assay buffer, $8 \mu \mathrm{L}$ DTNB solution, $2 \mu \mathrm{L} \mathrm{NADPH}$, and $10 \mu \mathrm{L}$ TrxR inhibitor or $10 \mu \mathrm{L}$ assay buffer.

\section{RESULTS}

\section{Full-length sequence and characterization of $\operatorname{SpTrx1}$}

The full-length cDNA of SpTrx1 was 672 bp, containing a 318-bp ORF (GenBank accession No. JQ864188.1), encoding a protein of 105 amino acids with a predicted molecular mass of $12.05 \mathrm{kDa}$, and an isoelectric point of 4.85. SpTrx 1 included a 5'-untranslated region (UTR) of $78 \mathrm{bp}$ and a 3'-UTR of $276 \mathrm{bp}$ including a poly (A) tail (Figure 1).

The amino acid sequence was aligned with those reported in other organisms (Figure 2). The SpTrx1 amino acid sequence shared the highest similarity with Trx1 from closely 
related species such as Portunus trituberculatus (86\%), Eriocheir sinensis (78\%), Fenneropenaeus chinensis (69\%), Daphnia pulex (68\%), Litopenaeus vannamei (67\%), Penaeus monodon (67\%), and Drosophila sechellia (63\%).

\begin{tabular}{|c|c|}
\hline & GACACGCTCTCCGTCTGATTCTGTGTGTGGTTGCCTTGTATCCTCTCTGCCTGTCACTAC \\
\hline & GGTCTACCAAGTGAAAGACAAGGATGACTTTGACAAGCAG \\
\hline 1 & $\begin{array}{llllllllllll}V & \mathrm{Y} & \mathrm{Q} & \mathrm{V} & \mathrm{K} & \mathrm{D} & \mathrm{K} & \mathrm{D} & \mathrm{D} & \mathrm{F} & \mathrm{D} & \mathrm{K}\end{array}$ \\
\hline & AGCTGGACAAAAGCTTGTAGTGGTGGACTTCTATGCCACCTGGTGTGGCCCC \\
\hline 15 & $\begin{array}{lllllllllllll}\mathrm{K} & \mathrm{L} & \mathrm{V} & \mathrm{V} & \mathrm{V} & \mathrm{D} & \mathrm{F} & \mathrm{Y} & \mathrm{A} & \mathrm{T} & \mathrm{W} & \mathrm{C} & \mathrm{G}\end{array}$ \\
\hline 81 & TGCAAGATGATTTCCCCCAAGATTCAGGAGATGAGTGAGCAGATGAGTGATGTTGTGTTC \\
\hline 35 & K I $\quad \mathrm{Q} \quad \mathrm{E} \quad \mathrm{M}$ \\
\hline 241 & CTGAAGGTGGACGTGGATGAGAGTGAGGAGGTTGCCATGGCCTATCAGGTGTCTTGCATG \\
\hline 55 & E E V A M A \\
\hline 301 & CCCACCTTCATTTTCATCAAGGAGGGAAAGAAAGTGGACAGTTTCTCAGGAGCAAGTGAA \\
\hline 75 & $\begin{array}{lllllllllll}I & K & E & G & K & K & V & D & S & F & S\end{array}$ \\
\hline 361 & GACAAGCTCCGTGAATTCATTGCAAGGCTGAAGTAATTGAAGGACAATGACTTGGTTTCC \\
\hline 95 & $\begin{array}{lllllllllllllllll}D & K & L & R & E & F & I & A & R & L & K\end{array}$ \\
\hline 421 & CTCAGGATCATCTCTAATCTCCACTAAAGAAAAATTCACACAGATTATAAAGACATTTGG \\
\hline 81 & CAGATGCCGTAGTTTGCCTCACAACCACACTCTCATTTGATTTGTGTAAACTTTTTTTTT \\
\hline 41 & AACGGTTTCATGAGGATGTTACAATGTTCAACTTGTGCCTGCAACTTTTTGTATTTTGAC \\
\hline 01 & TTGTTGCCTTTATTTTCATGTTTCAAGAAAAGAAGCTAATAAATAAAGCACCCCGAAAAAA \\
\hline & \\
\hline
\end{tabular}

Figure 1. Nucleotide (above) and deduced amino acid (below) sequences of the Trx 1 cDNA from Scylla paramamosain. The start (ATG) and stop (TAA) codons are underlined. The conserved residues of the Cys-Gly-Pro-Cys center are shaded.

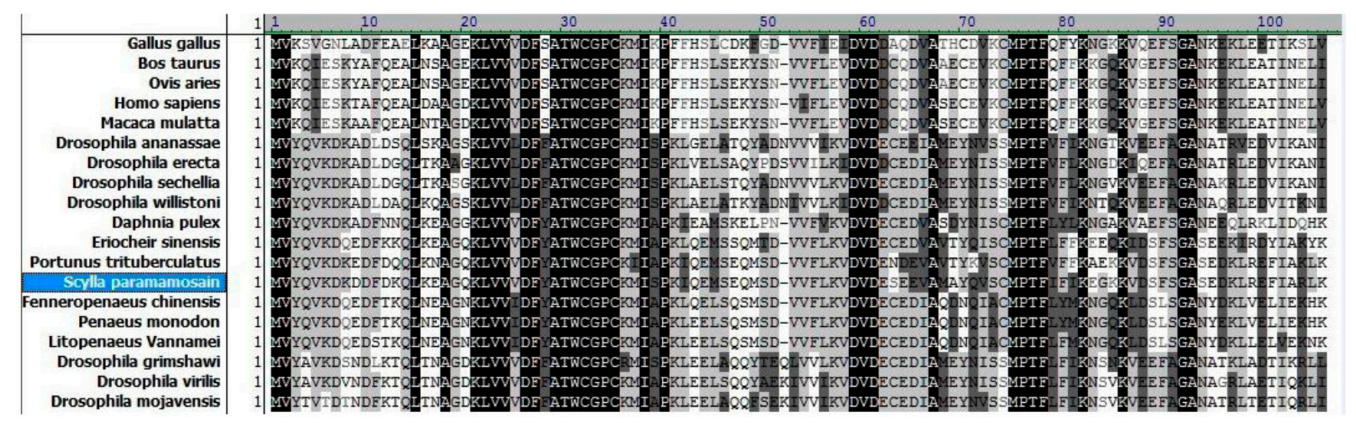

Figure 2. Multiple alignments of deduced SpTrx1 amino acid sequence and other Trx 1 amino acid sequences from NCBI using Vector NTI Suite 11.5. Gaps (-) were introduced to maximize the alignment. The relationships between residues are indicated as follows: non-similar residues, black letters on a white background; conserved residues, black letters on a light gray background; block of similarity, black letters on a dark gray background; identical residues, white letters on a black background; and weakly similar residues, light gray letters on a white background. All the proteins and their GenBank accession numbers are as follows: Gallus gallus (NP_990784.1), Bos taurus (NP_776393.1), Ovis aries (NP_001009421.1), Homo sapiens (NP_003320.2), Macaca mulatta (NP_001036197.2), Drosophila ananassae (XP_001964901.1), Drosophila erecta (XP_001969325.1), Drosophila sechellia (XP_002036354.1), Drosophila willistoni (XP_002064471.1), Daphnia pulex (EFX83063.1), Eriocheir sinensis (ACQ59118.1), Portunus trituberculatus (AFE88625.1), Scylla paramamosain (AGC96525.1), Fenneropenaeus chinensis (ACX30746.1), Penaeus monodon (ADV36299.1), Litopenaeus vannamei (ACA60746.1), Drosophila grimshawi (XP_001988235.1), Drosophila virilis (XP_002057775.1), Drosophila mojavensis (XP_002002787.1). 


\section{Phylogenetic tree of SpTrx1}

A neighbor-joining phylogenetic tree demonstrated the evolutionary relationships between various species (Figure 3). The analysis involved 25 amino acid sequences. All positions containing gaps and missing data were eliminated. A total of 105 positions were present in the final dataset. Evolutionary analyses were conducted in MEGA 5.0 (Tamura et al., 2011). According to the tree, the evolution of Trx was consistent with the conventional categories.

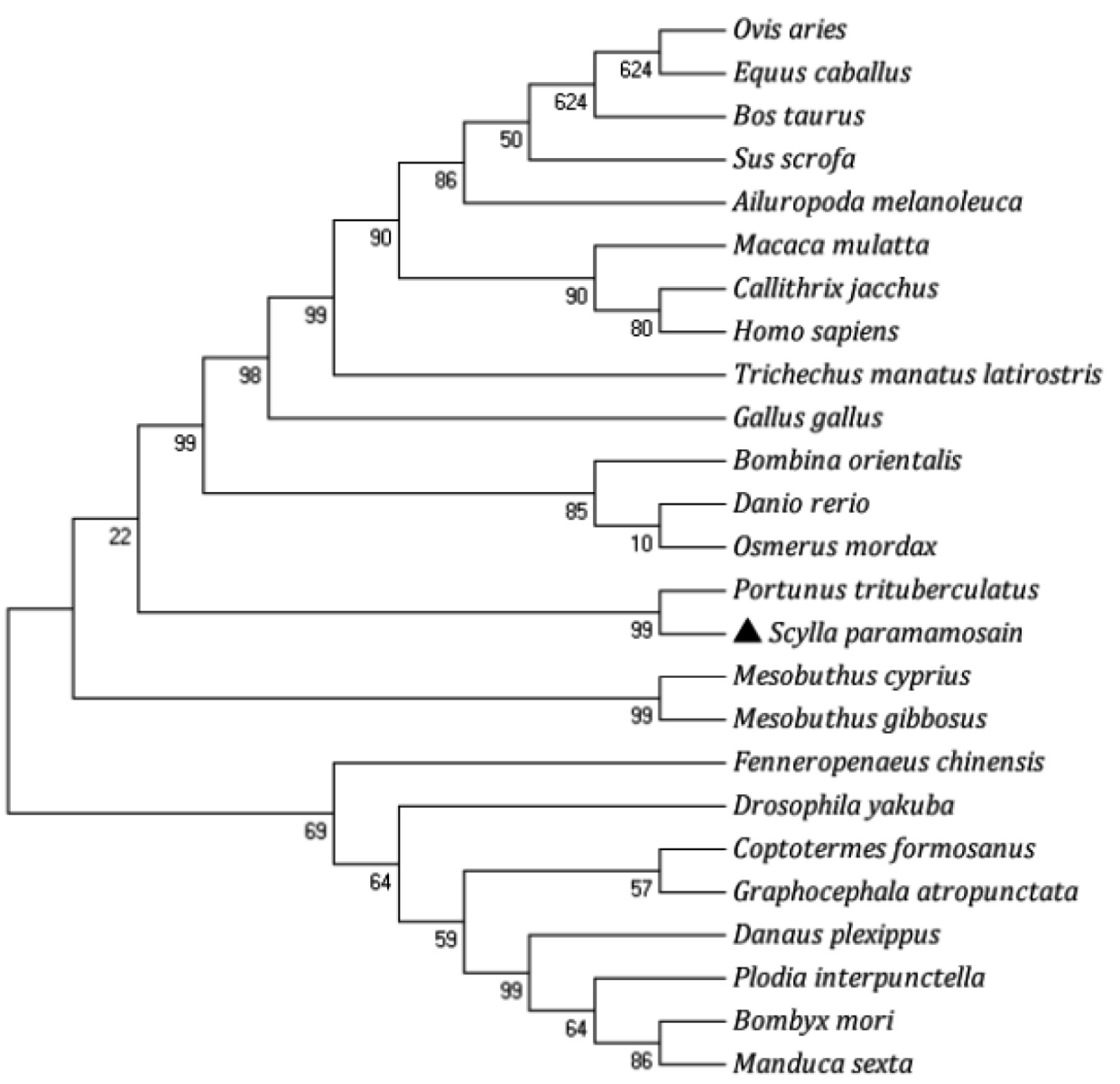

Figure 3. Evolutionary tree of thioredoxin-1 constructed by the neighbor-joining method. The scale bar corresponds to estimated amino acid substitutions per site. The sequences used were Ovis aries (NP_001009421.1), Equus caballus (NP 001075282.1), Bos taurus (NP 776393.1), Sus scrofa ( NP 999478.1), Ailuropoda melanoleuca (XP_002916571.1), Macaca mulatta ( NP_001036197.2), Callithrix jacchüs (XP_002743237.1), Homo sapiens (NP_003320.2), Trichechus manatus latirostris (XP_004372237.1), Gallus gallus (NP_990784.1), Bombina orientalis (ACJ12082.1), Danio rerio (NP_001002461.1), Osmerus mordax (ACO09971.1), Portunus trituberculatus (AFE88625.1), Scylla paramamosain (AGC96525.1), Mesobuthus cyprius (CAE54120.1), Mesobuthus gibbosus (CAE54157.1), Fenneropenaeus chinensis (ACX30746.1), Drosophila yakuba (XP_002100028.1), Coptotermes formosanus (AFZ78678.1), Graphocephala atropunctata (ABD98743.1), Danaus plexippus (EHJ64037.1), Plodia interpunctella (CBW45298.1), Bombyx mori (NP_001091804.1), Manduca sexta (AAF16695.1). 


\section{Protein structure of SpTrx1}

The secondary structural model of SpTrx 1 showed that $34.3 \%$ was alpha helixes, $27.7 \%$ was extended strands, and 39\% was loops. The 3-dimensional structure of SpTrx1 was submitted to the SWISS-MODEL server by searching PDB. A structure with $67.62 \%$ identity with Litopenaeus vannamei Trx (PDB ID: 3zzx, chain B, distance of $1.80 \AA$ ) was selected as the model. Although there were some differences in the Trx protein sequences, the spatial structures were highly conserved in various species (Figure 4).

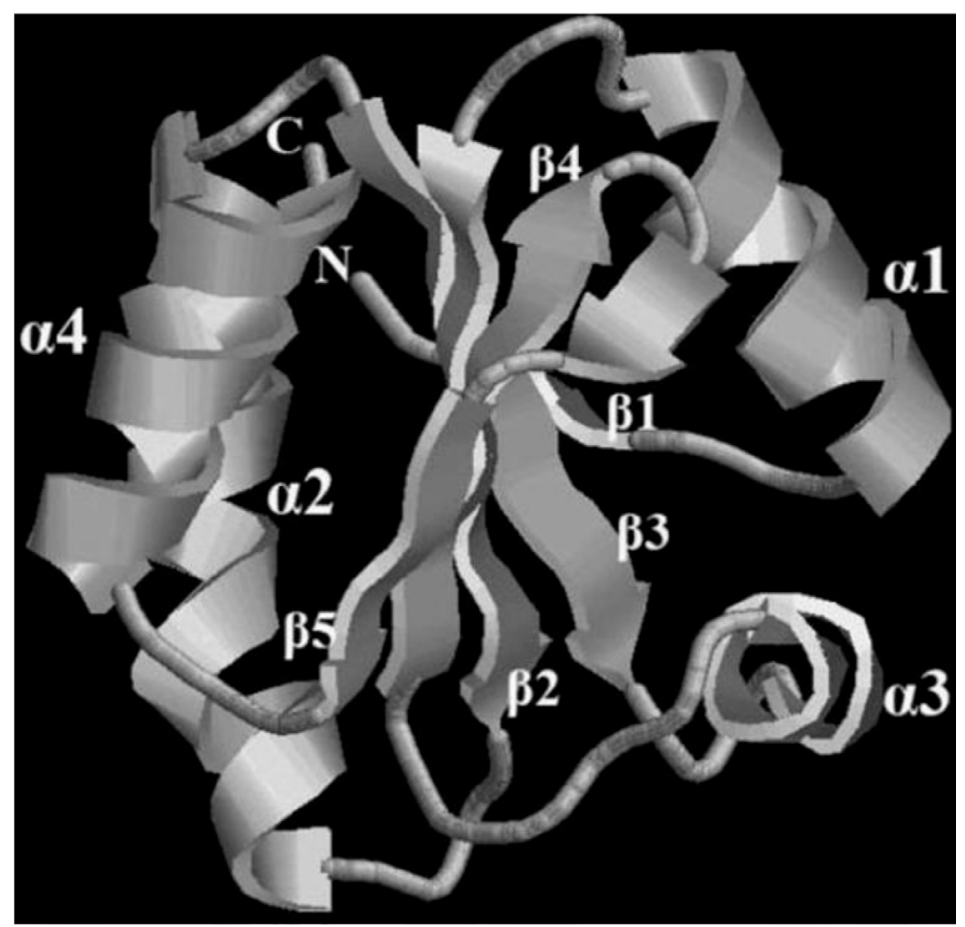

Figure 4. Predicted 3-D model of SpTrx1. It was based on Litopenaeus vannamei Trx1 available at PDB ID: 3zzx, chain B (established based on Swiss Model at the website (http://cn.expasy.org). The 3-D structure of the deduced SpTrx 1 contains $4 \alpha$-helices and $5 \beta$-sheets. " $N$ " indicates the $\mathrm{N}$-terminal residue. "C" indicates the C-terminal residue.

\section{Relative expression of $\operatorname{SpTrx1}$ in different tissues}

The relative expression of $\operatorname{Sp} \operatorname{Tr} x 1$ in the hepatopancreas, hemolymph, heart, muscle, testis, and gill was investigated using qRT-PCR. All data are reported in terms of relative mRNA expression as mean $\pm \mathrm{SD}$. The results were subjected to one-way analysis of variance (ANOVA) (SPSS 17.0). P values less than 0.05 and 0.01 were considered to be statistically significant and extremely significant, respectively. ANOVA indicated that there were significant differences in the relative expression of SpTrx1 in different tissues $(\mathrm{P}<0.05)$ : SpTrx 1 was expressed mainly in the testis, followed by the gill, then muscle and hepatopancreas, and finally the hemolymph (Figure 5). 


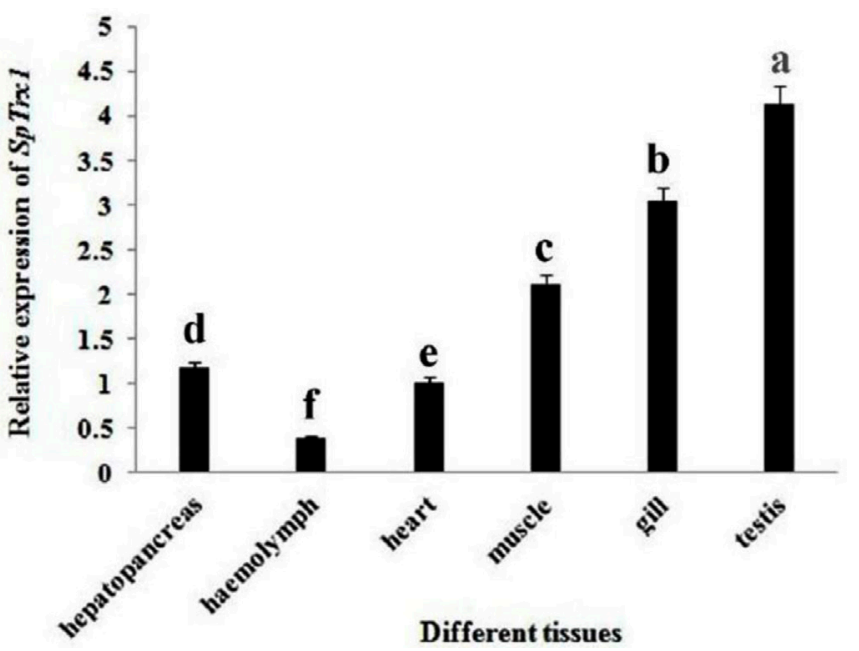

Figure 5. Expression profiles of the SpTrxl gene in different tissues as detected by quantitative real-time PCR. The amount of Trx 1 mRNA was normalized to the $18 S r R N A$ transcript level. Data are reported as means \pm SD of three independently repeated experiments. The y-axis represents the relative ratio of expression levels of $\operatorname{SpTrx} 1 / 18 S$ $r R N A$ mRNA.

\section{Relative expression of SpTrx1 from S. paramamosain injected with V.parahaemolyticus}

To examine the immune responses of $\operatorname{SpTrx} 1$ with an immune challenge, the relative expression of $\operatorname{SpTrxl}$ in hemolymph was measured after $V$. parahaemolyticus injection. The relative expression of the $\operatorname{SpTrxl}$ gene between the experimental groups and the control group did not show a significant difference within $4 \mathrm{~h}(\mathrm{P}>0.05)$ after treatment, and significant differences emerged 24 to $72 \mathrm{~h}$ after treatment $(\mathrm{P}<0.01)$. This revealed that the change in the temporal expression of the SpTrxl gene with V. parahaemolyticus challenge is not obvious in the beginning. Then, expression gradually increased, peaked at $72 \mathrm{~h}$ post-infection, and returned to the normal level. Actually, the lowest expression of $\operatorname{Sp} \operatorname{Tr} x 1$ was observed at $12 \mathrm{~h}$. The results showed that the relative expression level of SpTrxl in hemolymph with $V$. parahaemolyticus challenge significantly differed $(\mathrm{P}<0.05$; Figure 6), suggesting that $\operatorname{SpTr} x 1$ participated in the immune response of mud crabs.

\section{Determination of TrxR activity in $S$. paramamosain}

The standard curve equation of TrxR activity is $y=0.0158 x+0.043\left(R^{2}=0.9983\right)$, which has a single optical density (OD) value every 10 min that presented a linear relationship and could calculate the TrxR activity by the change in absorbance at $412 \mathrm{~nm}\left(\Delta \mathrm{A}_{412} \mathrm{~nm}\right)$. That is, the OD value of $T_{N B}^{2-}$ generated by TrxR and $\Delta A_{412} n m=\left(A_{2 t}-A_{11}\right)-\left(A_{2 I}-A_{11}^{41}\right)$. All data are reported in terms of the relative TrxR activity as means \pm SD. The results were subjected to one-way ANOVA (SPSS 17.0). P values less than 0.05 and 0.01 were considered to be statistically significant and extremely significant, respectively. ANOVA indicated that there were significant differences in different tissues $(\mathrm{P}<0.05)$. The TrxR activity was the highest in gill, second highest in heart, and lowest in muscle (Figure 7). The data showed that TrxR might mainly work on gill and heart in S. paramamosain. 


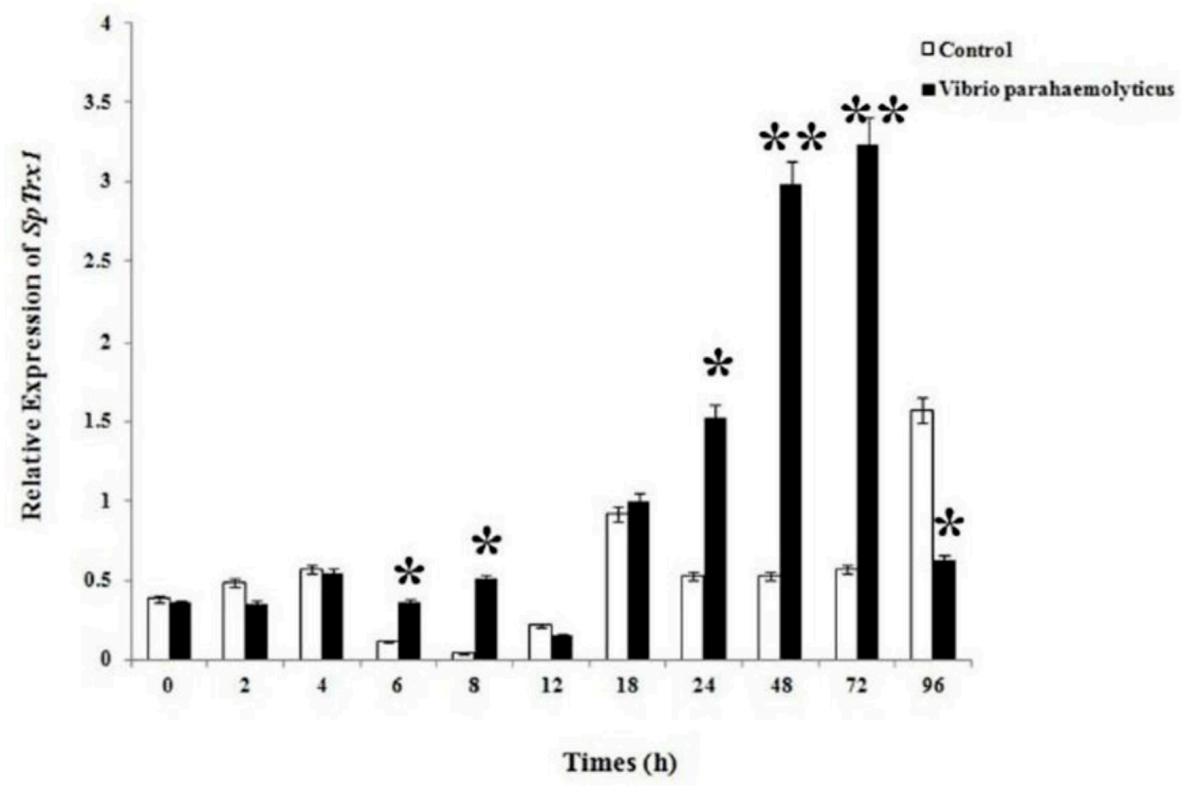

Figure 6. Temporal expression of $S p \operatorname{Tr} x 1$ mRNA relative to $18 S$ rRNA by quantitative real-time PCR in hemocytes after live Vibrio parahaemolyticus challenge. Significant differences across control $(0 \mathrm{~h})$ are indicated with an asterisk at $\mathrm{P}<0.05$, and with two asterisks at $\mathrm{P}<0.01$. Vertical bars are reported as means $\pm \mathrm{SD}$ of three independently repeated experiments.

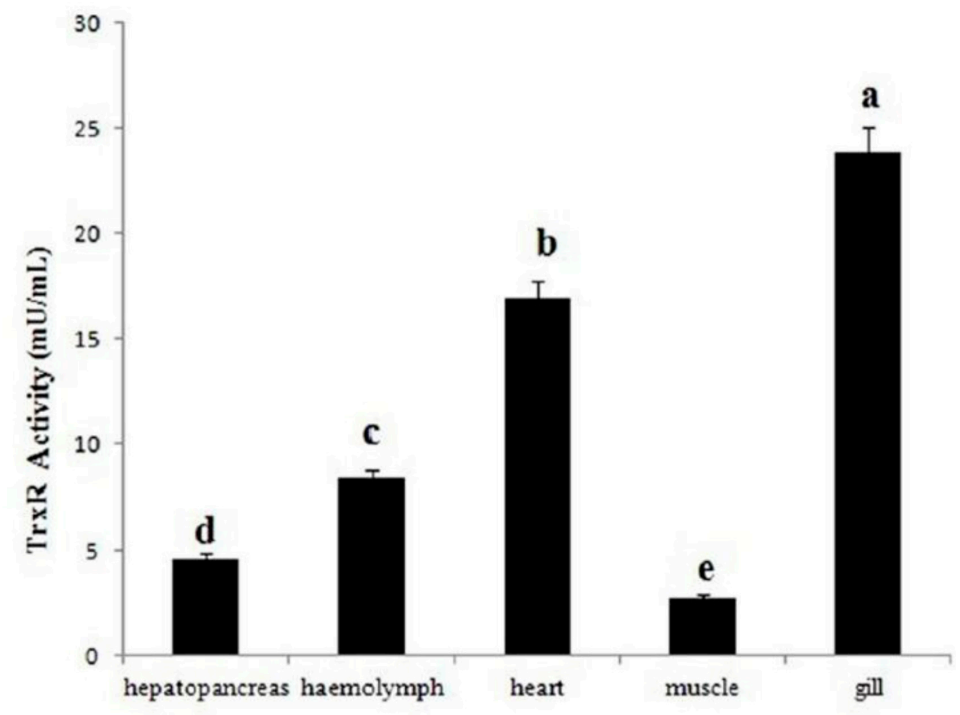

different tissue

Figure 7. Expression profiles of the TrxR activity in different tissues. The letters show that there were significant differences in different tissues (gill, heart, muscle, hemolymph, and hepatopancreas) $(\mathrm{P}<0.05)$. Data are reported as means $\pm \mathrm{SD}$ of three independently repeated experiments. 
To examine the immune responses of the TrxR activity against the immune challenge, the relative TrxR activity in different tissues (hemolymph, hepatopancreas, gill, and heart) was measured after $V$. parahaemolyticus challenge. The relative TrxR activity had the same tendency in each tissue $(\mathrm{P}<0.01)$. The TrxR activity went up $2 \mathrm{~h}$ after injection, peaked at $8 \mathrm{~h}$, slowly decreased from 12 to $24 \mathrm{~h}$, and finally returned to normal levels at $48 \mathrm{~h}$. Furthermore, TrxR activity followed a pattern that was similar to that of tissue expression in which the highest activity was in heart and the lowest was in hepatopancreas (Figure 8).

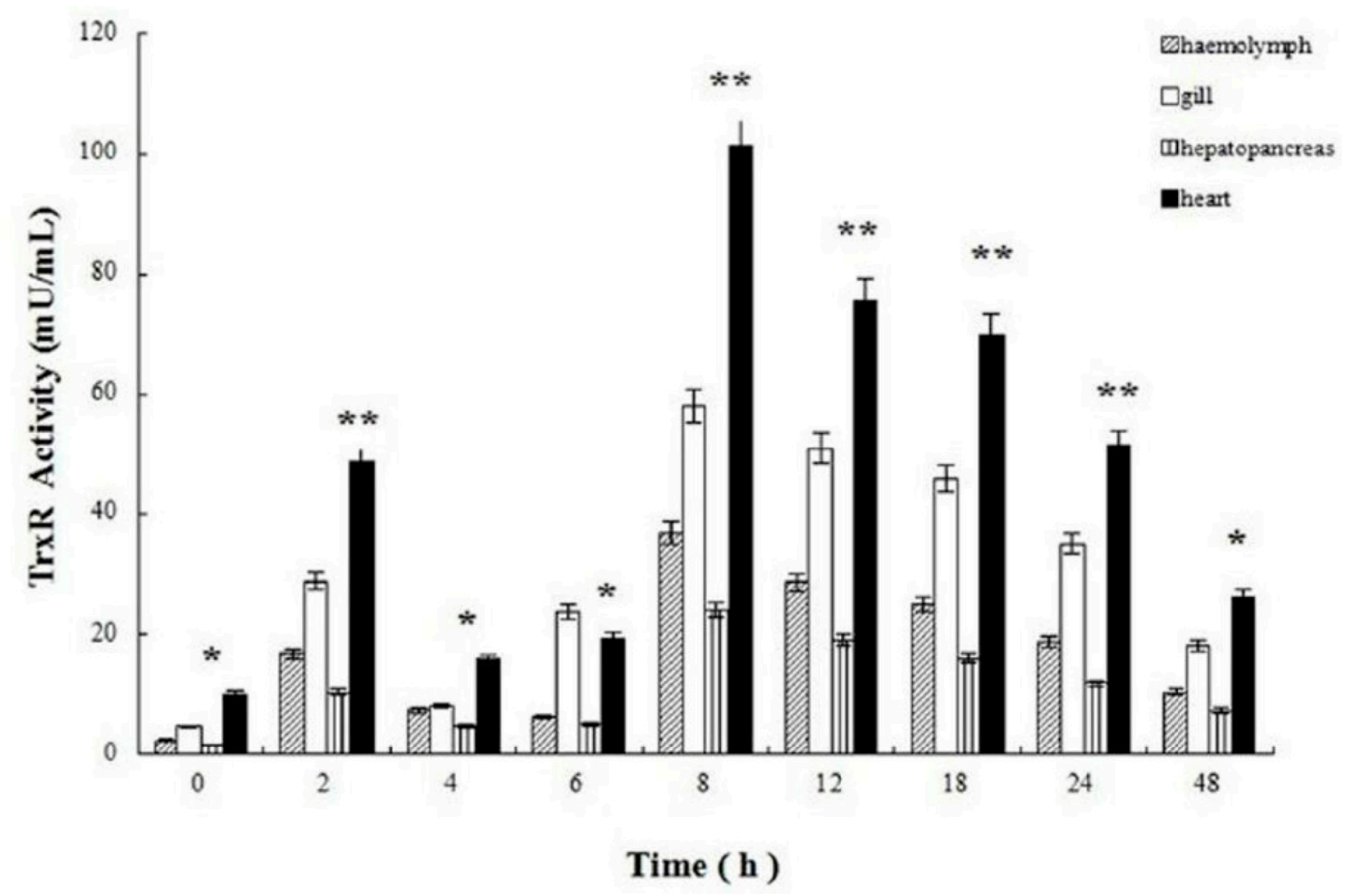

Figure 8. Temporal expression of TrxR activity in different tissues. Significant differences are indicated with an asterisk at $\mathrm{P}<0.05$, and with two asterisks at $\mathrm{P}<0.01$. Absolute black is the result of heart, absolute white is the result of gill, diagonal stripe is the result of haemolymph, and vertical stripe is the result of hepatopancreas. Vertical bars are reported as means \pm SD of three independently repeated experiments.

\section{DISCUSSION}

Trx 1 is ubiquitous among all living organisms and highly conserved at the amino acid sequence level. The CGPC motif in Trx 1 has been demonstrated to be ideally suited to control protein function via the redox state of structural or catalytic thiol groups (Powis and Montfort, 2001; Nkabyo et al., 2002). This study specifically confirmed that the typical active site sequence CGPC is highly conserved in SpTrx1. The most abundant amino acids in the SpTrx1 protein are lysine $(12.4 \%)$, valine $(11.4 \%)$, aspartic acid $(9.5 \%)$, glutamic acid $(8.6 \%)$, serine $(7.6 \%)$, and phenylalanine $(6.7 \%)$. Alignments and phylogenetic analysis were performed with several Trx sequences to obtain additional information (Figures 2 and 3). The amino acid sequence alignment showed that a putative polypeptide shared the highest similarity with Trx 1 from closely related species of Portunus trituberculatus (86\%). The phylogenetic tree dem- 
onstrated that Trx 1 amino acid sequences could reflect the evolutionary relationship among organisms clearly.

To further study the function of SpTrx1 and its secondary structure, a 3-dimensional model was established using the PredicProtein software and the SWISS-MODEL prediction algorithm. The secondary structure exhibited that SpTrx 1 contains $4 \alpha$-helices and $5 \beta$-sheets in the following order: $\beta 1-\alpha 1-\beta 2-\alpha 2-\beta 3-\alpha 3-\beta 4-\beta 5-\alpha 4$ (https://www.predictprotein.org/) (Schwede et al., 2003; Arnold et al., 2006), showing a high similarity with L. vannamei Trx1 ( $\beta 1-\alpha 1-\beta 2-$ $\alpha 2-\beta 3-\alpha 3-\beta 4-\beta 5-\alpha 4$ ) (Aispuro-Hernandez et al., 2008). It could be deduced from the secondary structure of SpTrx 1 that the conserved CGPC redox-active site is located at the end of $\beta 2$ and in the beginning of a long $\alpha$-helix ( $\alpha 2)$. These features are also similar to Trx1 from P. trituberculatus ( $\beta 1-\alpha 1-\beta 2-\alpha 2-\beta 3-\alpha 3-\beta 4-\beta 5-\alpha 4)$ (Song et al., 2012). Above all, their secondary structures are highly similar, and the oxidoreductase catalytic site and tautomerase motif are conserved; therefore, they may have the same function or have functional correlation.

The expression pattern of a gene is closely related to its function. In this study, we examined the relative quantities of $S p \operatorname{Tr} x 1$ in several tissues from healthy crabs by qRT-PCR and found that $\operatorname{SpTrx1}$ transcripts were expressed in gill, muscle, heart, hemolymph, testis, and hepatopancreas, which indicated that it could be potentially involved in some important cellular functions in $S$. paramamosain. The expression was the most abundant in testis and gill, and it was the lowest level in hemolymph. A previous study reported that $\operatorname{Tr} x 1$ transcripts of $P$. trituberculatus were mainly detected in gill, and the lowest detection was in muscle and stomach (Song et al., 2012). All results indicated that Trxl expression was high in the gill, suggesting that the gill is a possible route for the entry of pathogens and that Trx 1 may act to resist the invasion of pathogens. Therefore, these results may also serve as evidence to address the importance of the gill and hepatopancreas in the immune system of crab.

V. parahaemolyticus was first isolated in 1950, and it is now considered to be a major cause of gastroenteritis (Joseph et al., 1982; Janda et al., 1988). V. parahaemolyticus is also regarded as a major food-borne pathogen. In this study, V. parahaemolyticus was chosen to infect $S$. paramamosain. The temporal expression of the $S p \operatorname{Tr} x 1$ gene increased gradually and peaked at $72 \mathrm{~h}$ post-infection. However, Song et al. (2012) reported that after $V$. alginolyticus challenge, Trx 1 of $P$. trituberculatus mRNA expression was slightly increased compared with that in the corresponding control group, and then it was upregulated markedly at $48 \mathrm{~h}$ post infection, reaching a maximum level at $72 \mathrm{~h}$ post-infection in $P$. trituberculatus. Above all, the change in Trx $1 \mathrm{mRNA}$ expression in S. paramamosain is similar to that in P. trituberculatus, with the highest expression at $72 \mathrm{~h}$. This result indicated that Trx 1 was perhaps involved in the responses to bacterial challenge. This point requires further study.

In this experiment, TrxR catalyzes the reduction of DTNB with NADPH to TNB ${ }^{2-}$, which generates a strong yellow color. Because other enzymes can also reduce DTNB in crude biological samples, such as glutathione reductase and glutathione peroxidase, a TrxR-specific inhibitor is utilized to determine the TrxR-specific activity. The results showed that TrxR activity was the highest in gill, which proved that the gill may be an important immune organ in $S$. paramamosain. TrxR activity was relatively stable in $S$. paramamosain injected with $V$. parahaemolyticus in hemolymph, hepatopancreas, gill, and heart.

However, the relevance of the relationship between TrxR activity and mRNA expression in the Trx system is not entirely clear. TrxR is an NADPH-dependent oxidoreductase. Together with Trx (Arnér et al., 1999; Gromer et al., 2004), which catalyzes the reduction of the disulfide bridge in thioredoxin by NADPH, TrxR was purified from calf liver and thymus 
(Holmgren, 1977). To be active, Trx must be in the reduced form, and this state is maintained by the flavoenzyme TrxR at the expense of the reducing power of NADPH (Holmgren and Björnstede, 1995). The functions ascribed to Trxs are continuously increasing because they were initially discovered as electron donors for ribonucleotide reductase, an essential enzyme in DNA synthesis (Laurent et al., 1964).

We found that both TrxR activity and mRNA relative expression are high in gill (Figures 5 and 7), which could demonstrate that $\operatorname{SpTr} x 1$ mRNA relative expression was similar to TrxR activity. On the other hand, SpTrx 1 mRNA relative expression was different from TrxR activity with $V$. parahaemolyticus challenge in hemolymph (Figures 6 and 9). mRNA relative expression was higher at 24 and $48 \mathrm{~h}$, but TrxR activity was higher from 8 to $18 \mathrm{~h}$. This difference may be because TrxR activity is affected by other factors. In the DTNB reduction assay TrxR activity was determined by the NADPH-dependent reduction of DTNB (Arnér et al., 1999). TrxR activity was also assayed based on the ability of TrxR to reduce Trx, which then reduces insulin disulfide bridges (Holmgren, 1979). Besides, TrxR inhibitors would be promising agents against the insect stages of the parasite and could also be used as insecticides. This perspective is supported by the fact that there are great structural and functional differences among the Trx-reducing centers of TrxR in insects, malarial parasites, and mammals (Kanzok et al., 2000).

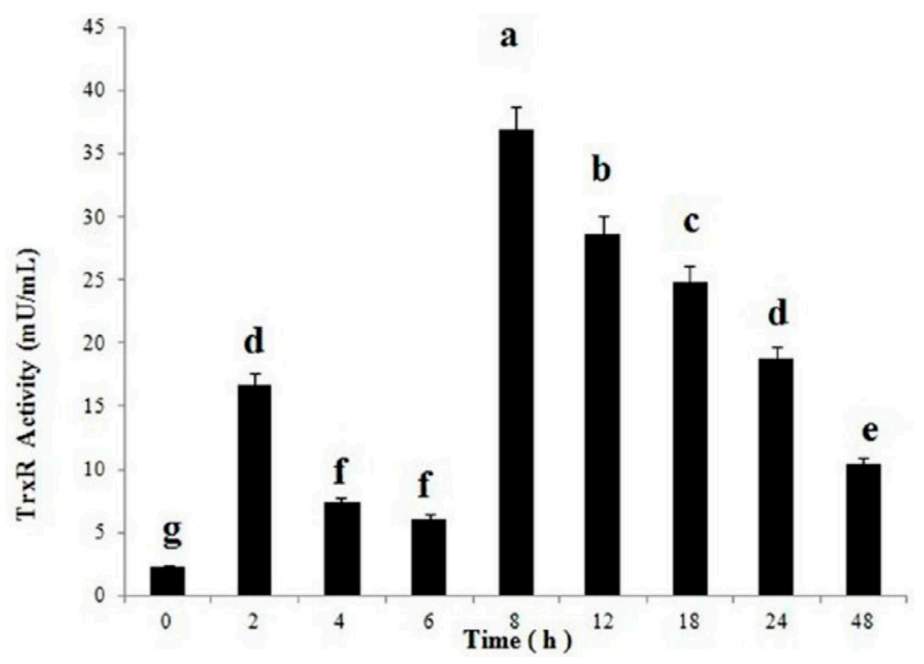

Figure 9. TrxR activity in hemolymph challenged by Vibrio parahaemolyticus. Significant differences are indicated with an asterisk at $\mathrm{P}<0.05$, and with two asterisks at $\mathrm{P}<0.01$. Vertical bars are reported as means $\pm \mathrm{SD}$ of three independently repeated experiments.

According to our results, $\operatorname{Tr} x 1$ may be a potential biomarker gene to evaluate environmental stress in aquatic organisms. Trx was closely related to TrxR in the Trx system. Further studies are needed to better understand the controlling mechanisms of Trx in the immune system. A functional study of SpTrxl in the crab immune system may be applied to aquaculture production. According to these studies, we look forward to boosting the immunity of crabs, strengthening the property, and reducing the use of antibiotics, thereby improving aquaculture production and quality. 


\section{ACKNOWLEDGMENTS}

Research supported by the National Natural Science Foundation of China (\#31101890), the Science and Technology Commission of Shanghai Municipality (\#10JC1418600), and the Basic Research Fund for State-Level Nonprofit Research Institutes of ESCFRI and CAFS (\#2012T05).

\section{REFERENCES}

Aispuro-Hernandez E, Garcia-Orozco KD, Muhlia-Almazan A, Del-Toro-Sanchez L, et al. (2008). Shrimp thioredoxin is a potent antioxidant protein. Comp. Biochem. Physiol. C Toxicol. Pharmacol. 148: 94-99.

Arnér ES, Zhong LW and Holmgren A (1999). Preparation and assay of mammalian thioredoxin and thioredoxin reductase. Methods Enzymol. 300: 226-239.

Arnold K, Bordoli L, Kopp J and Schwede T (2006). The SWISS-MODEL workspace: a web-based environment for protein structure homology modelling. Bioinformatics 22: 195-201.

Collet JF, D'Souza JC, Jakob U and Bardwell JCA (2003). Thioredoxin 2, an oxidative stress-induced protein, contains a high affinity zinc binding site. J. Biol. Chem. 278: 45325-45332.

Dopazo J (1994). Estimating errors and confidence intervals for branch lengths in phylogenetic trees by a bootstrap approach. J. Mol. Evol. 38: 300-304.

Gromer S, Urig S and Becker K (2004). The thioredoxin system - from science to clinic. Med. Res. Rev. 24: 40-89.

Holmgren A (1977). Bovine thioredoxin system. Purification of thioredoxin reductase from calf liver and thymus and studies of its function in disulfide reduction. J. Biol. Chem. 252: 4600-4606.

Holmgren A (1979). Thioredoxin catalyzes the reduction of insulin disulfides by dithiothreitol and dihydrolipoamide. $J$. Biol. Chem. 254: 9627-9632.

Holmgren A (1985). Thioredoxin. Annu. Rev. Biochem. 54: 237-271.

Holmgren A and Björnstede M (1995). Thioredoxin and thioredoxin reductase. Methods Enzymol. 252: 199-208.

Imai H and Takeda M (2005). A natural hybrid mud crab (Decapoda, Portunidae) from Japan. J. Crustacean Biol. 25: 620-624.

Imjongjirak C, Amparyup P, Tassanakajon A and Sittipraneed S (2007). Antilipopolysaccharide factor (ALF) of mud crab Scylla paramamosain: molecular cloning, genomic organization and the antimicrobial activity of its synthetic LPS binding domain. Mol. Immunol. 44: 3195-3203.

Janda JM, Powers C, Bryant RG and Abbott SL (1988). Current perspectives on the epidemiology and pathogenesis of clinically significant Vibrio spp. Clin. Microbiol. Rev. 1: 245-267.

Jiang KJ, Zhang FY, Zhang D, Tao QC, et al. (2011). Identification of a trypsin gene from Scylla paramamosain and its expression profiling during larval development. Afr. J. Agr. Res. 6: 6613-6621.

Jin J, Chen XH, Zhou Y, Bartlam M, et al. (2002). Crystal structure of the catalytic domain of a human thioredoxin-like protein: implications for substrate specificity and a novel regulation mechanism. Eur. J. Biochem. 269: 2060-2068.

Joseph SW, Colwell RR and Kaper JB (1982). Vibrio parahaemolyticus and related halophilic Vibrios. Crit. Rev. Microbiol. 10: 77-124.

Kanzok SM, Schirmer RH, Türbachova I, Iozef R, et al. (2000). The thioredoxin system of the malaria parasite Plasmodium falciparum. Glutathione reduction revisited. J. Biol. Chem. 275: 40180-40186.

Keenan CP, Davie PJF and Mann DL (1998). A revision of the genus Scylla De Haan, 1833 (Crustacea: Decapoda: Brachyura: Portunidae). Raffles B. Zool. 46: 217-245.

Khayat M, Stuge TB, Wilson M, Bengtén E, et al. (2001). Thioredoxin acts as a B cell growth factor in channel catfish. $J$. Immunol. 166: 2937-2943.

Kim DH, Kim JW, Jeong JM, Park HJ, et al. (2011). Molecular cloning and expression analysis of a thioredoxin from rock bream, Oplegnathus fasciatus, and biological activity of the recombinant protein. Fish Shellfish Immunol. 31: 22-28.

Kondo N, Nakamura H, Masutani H and Yodoi J (2006). Redox regulation of human thioredoxin network. Antioxid. Redox Signal. 8: 1881-1890.

Laurent TC, Moore EC and Reichard P (1964). Enzymatic synthesis of deoxyribonucleotides. IV. Isolation and characterization of thioredoxin, the hydrogen donor from Escherichia coli B. J. Biol. Chem. 239: 3436-3444.

Matsui M, Oshima M, Oshima H, Takaku K, et al. (1996). Early embryonic lethality caused by targeted disruption of the mouse thioredoxin gene. Dev. Biol. 178: 179-185.

Matthews JR, Wakasugi N, Virelizier JL, Yodoi J, et al. (1992). Thioredoxin regulates the DNA binding activity of NF- $\mathrm{kB}$ 
by reduction of disulfide bond involving cysteine 62. Nucleic Acids Res. 20: 3821-3830.

Mu CK, Zhao JM, Wang LL, Song LS, et al. (2009). A thioredoxin with antioxidant activity identified from Eriocheir sinensis. Fish Shellish Immunol. 26: 716-723.

Müller S, Gilberger TW, Krnajski Z, Lüersen K, et al. (2001). Thioredoxin and glutathione system of malaria parasite Plasmodium falciparum. Protoplasma 217: 43-49.

Nkabyo YS, Ziegler TR, Gu LH, Watson WH, et al. (2002). Glutathione and thioredoxin redox during differentiation in human colon epithelial (Caco-2) cells. Am. J. Physiol. Gastrointest. Liver Physiol. 283: G1352-G1359.

Nordberg J and Arnér ES (2001). Reactive oxygen species, antioxidants, and the mammalian thioredoxin system. Free Radic. Biol. Med. 31: 1287-1312.

Powis G and Montfort WR (2001). Properties and biological activities of thioredoxins. Annu. Rev. Pharmacol. Toxicol. 41: 261-295.

Rodriguez J, Boulo V, Mialhe E and Bachere E (1995). Characterisation of shrimp haemocytes and plasma components by monoclonal antibodies. J. Cell Sci. 108: 1043-1050.

Rzhetsky A and Nei M (1992). A simple method for estimating and testing minimum-evolution trees. Mol. Biol. Evol. 9: 945-967.

Saitou N and Nei M (1987). The neighbor-joining method: a new method for reconstructing phylogenetic trees. Mol. Biol. Evol. 4: 406-425.

Schwede T, Kopp J, Guex N and Peitsch MC (2003). SWISS-MODEL: an automated protein homology-modeling server. Nucleic Acids Res. 31: 3381-3385.

Song CW, Cui ZX, Liu Y, Wang SY, et al. (2012). First report of two thioredoxin homologues in crustaceans: molecular characterization, genomic organization and expression pattern in swimming crab Portunus trituberculatus. Fish Shellfish Immunol. 32: 855-861.

Song JY and Roe JH (2008). The role and regulation of Trx1, a cytosolic thioredoxin in Schizosaccharomyces pombe. J. Microbiol. 46: 408-414.

Song JY, Cha J, Lee J and Roe JH (2006). Glutathione reductase and a mitochondrial thioredoxin play an overlapping role for maintaining iron-sulfur enzymes in fission yeast. Eukaryot. Cell 5: 1857-1865.

Stoyanovsky DA, Tyurina YY, Tyurin VA, Anand D, et al. (2005). Thioredoxin and lipoic acid catalyze the denitrosation of low molecular weight and protein S-nitrosothiols. J. Am. Chem. Soc. 127: 15815-15823.

Tamura K, Peterson D, Peterson N, Stecher G, et al. (2011). MEGA5: molecular evolutionary genetics analysis using maximum likelihood, evolutionary distance, and maximum parsimony methods. Mol. Biol. Evol. 28: 2731-2739.

Yamawaki H, Haendeler J and Berk BC (2003). Thioredoxin: a key regulator of cardiovascular homeostasis. Circ. Res. 93: 1029-1033.

Zhang D, Jiang KJ, Zhang FY, Ma CY, et al. (2011). Isolation and characterization of a ferritin cDNA from the mud crab Scylla paramamosain. J. Crustacean Biol. 31: 345-351. 